\title{
Дискриминация поперечных мод в торцевых полупроводниковых лазерах с пространственной модуляцией отражения выходных зеркал
}

\author{
(ㄱ Н.Ю. Гордеев ${ }^{1}$, А.С. Паюсов ${ }^{1}$, И.С. Мухин ${ }^{2,3}$, А.А. Серин ${ }^{1}$, М.М. Кулагина ${ }^{1}$, Ю.А. Гусева ${ }^{1}$, \\ Ю.М. Шерняков ${ }^{1}$, Ю.М. Задиранов ${ }^{1}$, М.В. Максимов ${ }^{2}$ \\ ${ }^{1}$ Физико-технический институт им. А.Ф. Иоффре Российской академии наук, \\ 194021 Санкт-Петербург, Россия \\ ${ }^{2}$ Санкт-Петербургский национальный исследовательский Академический университет Российской академии наук, \\ 194021 Санкт-Петербург, Россия \\ ${ }^{3}$ Университет ИТМО, \\ 197101 Санкт-Петербург, Россия \\ E-mail: gordeev@switch.ioffe.ru
}

(Получена 1 августа 2018 г. Принята к печати 13 августа 2018 г.)

Разработана постростовая технология пространственной модуляции отражения выходных зеркал торцевых полупроводниковых лазеров, основанная на травлении диэлектрических покрытий фокусированным ионным пучком. Показано, что в лазерах на InAs/InGaAs-квантовых точках с шириной полоскового волновода 10 мкм модуляция отражения позволяет эффективно подавлять генерацию поперечных горизонтальных мод высокого порядка.

DOI: $10.21883 /$ FTP.2019.02.47100.8971

\section{1. Введение}

Максимальная мощность излучения торцевого полупроводникового лазера, ограниченная тепловым загибом ватт-амперной характеристики либо катастрофическим разрушением выходного зеркала, возрастает при увеличении ширины его полоска. В то же время увеличение ширины полоска лазера с гребневым волноводом приводит к возможности возбуждения поперечных горизонтальных мод (lateral modes) более высоких порядков. При многомодовом режиме излучения каждая мода имеет свою характерную диаграмму направленности и свое фокусное расстояние, что сильно ухудшает качество лазерного пучка. Для увеличения мощности одномодового излучения можно использовать лазер с переменной шириной полоска [1], в котором узкая часть полоска задает одномодовый характер излучения, а расширение полоска обеспечивает увеличение оптической мощности. Одномодовый характер излучения в расширенном полоске может обеспечиваться введением различными методами дополнительных оптических потерь для поперечных мод высоких порядков. Например, в работе [2] такая модовая селекция обеспечивалась за счет использования разрезного полоска. Развитием этого направления является конструкция лазера с интегрированным в полосок дифракционным фильтром мод высокого порядка [3]. Эти подходы объединяет то, что селекция поперечных мод осуществляется за счет введения дополнительных элементов вдоль полоскового волновода, усложняющих конструкцию лазера и негативно влияющих на параметры фундаментальной моды.

Подавляющее большинство полупроводниковых лазеров имеет выходные зеркала, полученные скалыванием кристалла по кристаллографическим плоскостям. На поверхность далее могут напыляться пассивирующие, просветляющие или отражающие покрытия. В любом из этих вариантов коэффициент отражения не зависит от координаты по поверхности зеркала. В то же время поперечные оптические моды имеют разное пространственное распределение интенсивности на лазерных зеркалах. Это создает принципиальную возможность дифференцированного изменения внешних оптических потерь для мод разного порядка при использовании лазерного зеркала с поверхностно неоднородным коэффициентом отражения [4]. Технологии напыления тонких пленок через маску позволяют создавать на лазерных зеркалах элементы с характерными размерами в десятки микрон, что было использовано в работе [5] для пространственной модуляции коэффициента отражения лазеров с широким полоском (broad-area laser), в работе [6] - для полоска 25 мкм. Альтернативным подходом является прецизионное травление лазерных зеркал или покрытий зеркал, например, фокусированным ионным пучком (ФИП) (focused ion beam, FIB) [7]. Идея была экспериментально реализована для модификации модового состава полосковых лазеров среднего ИК-диапазона [8]. Технологически метод состоял в напылении на зеркало диэлектрической пленки и слоя золота и последующего вытравливания ионным пучком окон в этом комбинированном покрытии. Существенным недостатком подхода является невысокая точность контроля глубины травления и появление оптических дефектов в полупроводниковом зеркале за счет треков торможения тяжелых ионов галлия [7].

В настоящей работе мы представляем результаты по разработке постростовой технологии пространственной 
модуляции коэффициента отражения выходных зеркал лазеров, основанной на травлении диэлектрических покрытий фокусированным ионным пучком. Метод использован для селекции поперечных горизонтальных оптических мод лазеров на InAs/InGaAs-квантовых точках с шириной полоскового волновода 10 мкм.

\section{2. Модель лазера с пространственной модификацией оптических потерь на выход}

Для подавления горизонтальных мод высокого порядка в торцевых диодных лазерах нами была разработана численная модель, основанная на следующих базовых принципах. Как известно, моды разных порядков имеют разное пространственное распределение интенсивности на лазерных зеркалах. На рис. 1 представлено схематическое изображение торца лазера с полосковым волноводом шириной $W$, который поддерживает две горизонтальные моды. В стандартном варианте коэффициенты отражения на зеркале для мод разного порядка практически одинаковы. Незначительная разница возникает из-за разных эффективных показателей преломления.

Коэффициент отражения на зеркалах определяет оптические потери на выход излучения из лазерного резонатора. Для моды $n$ внешние потери будут определяться по известной формуле $\alpha_{n}=(1 / 2 L) \ln \left(1 / R_{n} R\right)$, где $L-$ длина резонатора, $R_{n}$ и $R-$ коэффициенты отражения на зеркалах. В формуле коэффициент $R$ одного из зеркал принят одинаковым для всех мод, а коэффициент $R_{n}$ может отличаться для мод разного порядка. Из этой формулы легко вывести разницу внешних потерь для нулевой моды и моды порядка $m$ :

$$
\alpha_{m}-\alpha_{0}=(1 / 2 L) \ln \left(R_{0} / R_{m}\right),
$$

где $R_{0}$ и $R_{m}$ - коэффициенты отражения нулевой моды и моды порядка $m$ соответственно. Из этого

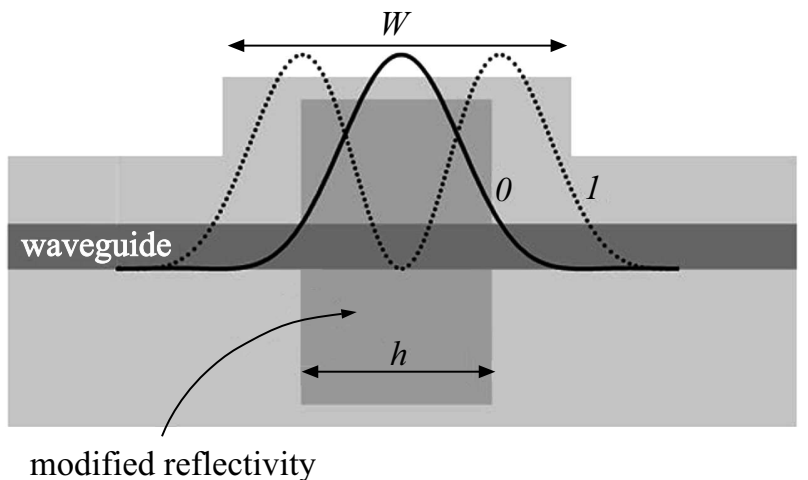

Рис. 1. Схематическое изображение зеркала торцевого лазера с полосковым волноводом с пространственной модификацией коэффициента отражения и профили интенсивности нулевой (сплошная линия) и первой (пунктирная линия) горизонтальных поперечных мод.

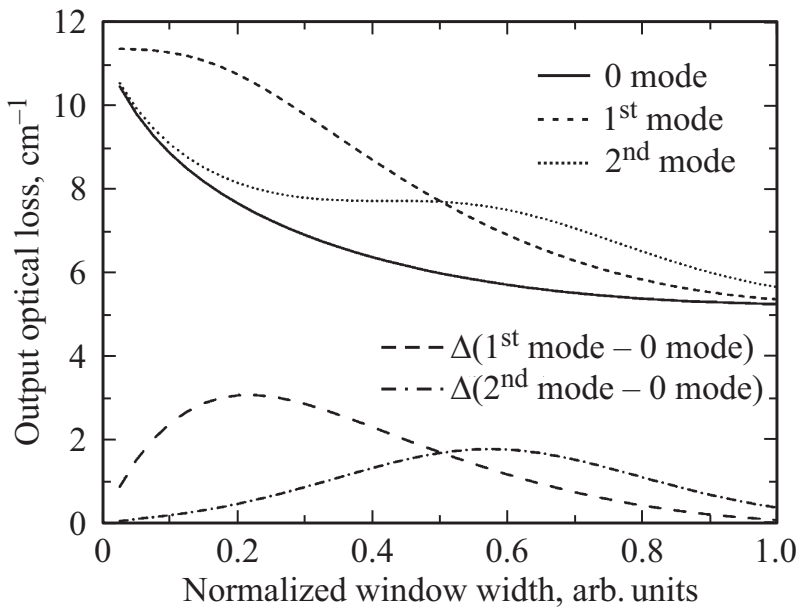

Рис. 2. Зависимость внешних потерь и разностных потерь для нулевой (фундаментальной), первой и второй мод модельного лазера в зависимости от нормированной ширины окна.

выражения видно, что для преимущественной генерации фундаментальной моды ее коэффициент отражения $R_{0}$ должен быть больше, чем для $m$ моды. Если пространственная локализация мод на зеркале отличается, то разница внешних потерь может быть обеспечена двумя способами: либо в области локализации нулевой моды на зеркале находится диэлектрическое отражающее покрытие (типичный коэффициент отражения более 95\%), либо в области локализации $m$ моды на зеркале находится просветляющее покрытие (типичный коэффициент отражения менее 5\%). Коэффициент отражения полупроводникового $\mathrm{AlGaAs-зеркала} \mathrm{составляет}$ порядка $35 \%$, поэтому максимальная разница $\alpha_{m}-\alpha_{0}$ в первом варианте пропорциональна $\ln (95 / 35)$, во втором - $\ln (35 / 5)$. Эта простая оценка показывает, что второй способ предпочтительнее, поскольку комбинация „чистый полупроводник-просветляющее покрытие“ дает больший контраст по внешним потерям, чем комбинация „чистый полупроводник-отражающее покрытие“.

Предположим, что на выходное зеркало лазера (рис. 1) нанесено просветляющее покрытие с коэффициентом отражения $R^{*}$. В центральной части гребневого волновода $W$ оставлено окно без покрытия шириной $h$, имеющее коэффициент отражения $R$. В этом случае коэффициент отражения $R_{n}$ на зеркале для оптической моды $n$ будет определяться как [6]

$$
R_{n}=R \cdot \Gamma_{n}+R^{*}\left(1-\Gamma_{n}\right),
$$

где $\Gamma_{n}$ - доля интенсивности моды $n$ на зеркале, находящаяся в области с коэффициентом отражения $R$. В вертикальном направлении оптические моды имеют практически одинаковый размер, поэтому на коэффициент отражение влияет только ширина окна $h$.

На рис. 2 представлены расчетные внешние потери и разностные потери для нулевой, первой и второй горизонтальных мод полоскового лазера в зависимости 
от нормированной ширины окна в напыленном просветляющем покрытии (коэффициент отражения 1\%). В расчете моды принимались гауссовой и ГауссаЭрмита соответственно. Для численной оценки длина резонатора была взята 2 мм, коэффициент отражения $R$ немодифицированного зеркала $35 \%$. Из зависимости видно, что разностные внешние потери могут достигать величины $3 \mathrm{~cm}^{-1}$, а сама зависимость является плавной, что позволяет уменьшить эффекты, связанные с уходами фактических размеров вытравленных окон от номинальных. При ширине окна 0.5 внешние оптические потери для первой и второй мод превышают потери для фундаментальной моды на $1.7 \mathrm{~cm}^{-1}$, что должно быть достаточно для подавления лазерной генерации на этих модах. В этом расчете мы ограничились рассмотрением полосковых волноводов, поддерживающих лазерную генерацию на трех горизонтальных модах. Отметим, что для селекции большого числа мод требуется модуляция коэффициента отражения, имеющая геометрию более сложную, чем рассматриваемая в данной работе.

\section{3. Изготовление и исследование лазерных диодов с пространственной модификацией оптических потерь на выход}

Для экспериментального исследования пространственной модификации внешних оптических потерь нами были изготовлены лазеры с активной областью на основе 10 слоев InAs/InGaAs-квантовых точек, излучающих на длине волны вблизи 1.3 мкм [9], с шириной полоскового волновода 10 мкм. При таких параметрах лазер не является одномодовым и имеет как минимум три поперечные горизонтальные моды. В качестве просветляющего покрытия использовалась диэлектрическая пленка $\mathrm{Ta}_{2} \mathrm{O}_{3}$ толщиной $157 \mathrm{Hм}(\lambda / 4)$, имеющая коэффициент отражение менее $2 \%$, наносимая методом плазменно-химического осаждения из газовой фазы.

Принципиально существует два варианта получения пространственной модификации коэффициента отражения: напыление диэлектрической пленки на лазерное зеркало через специальную маску для создания необходимого рисунка или пространственное селективное травление уже нанесенной пленки. Первый вариант технологически осуществим, но его большим недостатком является невозможность формирования рисунка микронного масштаба. Кроме того, негативно влияют эффекты подпыления на границах маски [5] и плохой контроль толщины наносимой пленки. Стандартной технологией для изготовления полупроводниковых приборов из эпитаксиальных пластин является травление с использованием методов фотолитографии, применение которых к отдельным лазерным кристаллам представляется малоосуществимым. По этим причинам нами была выбрана технология травления напыленного диэлектрического просветляющего покрытия фокусированным ионным

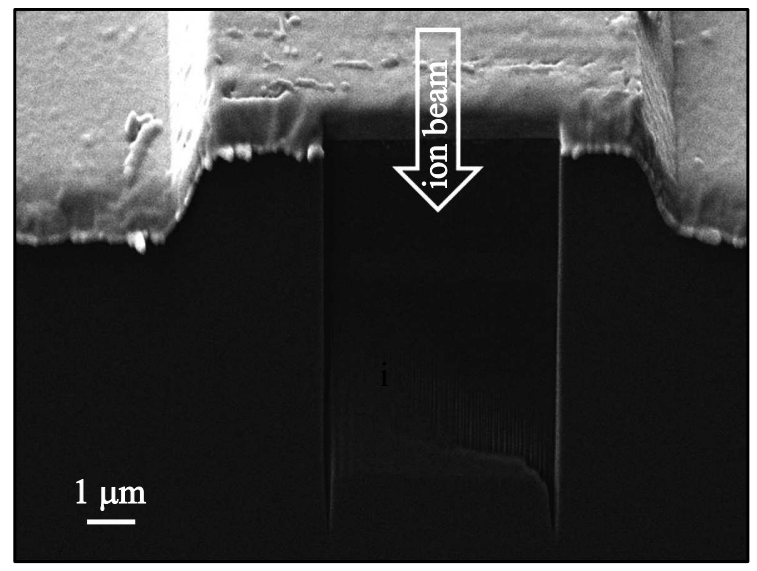

Рис. 3. Окно, вытравленное фокусированным ионным пучком в диэлектрическом просветляющем покрытии, нанесенном на зеркало торцевого лазера с шириной полоскового волновода 10 мкм. Стрелкой показано направление ионного пучка.

пучком [7]. Комплекс ФИП позволяет модифицировать структуры с нанометровым пространственным разрешением. В установке ФИП локальная обработка структур сфокусированным потоком ионов галлия имеет in situ контроль процесса травления, обеспечиваемый сканирующим электронным микроскопом (СЭМ). Технология позволяет создавать структуры не только простых геометрических форм (прямоугольник, круг, линия, точка), но также проводить обработку по заранее заданному алгоритму.

Для сохранения качества полупроводникового лазерного зеркала мы использовали травление пучком, параллельным плоскости зеркала (рис. 3). В такой геометрии ионы галлия тормозятся в диэлектрической пленке в области, лежащей существенно ниже волноводных слоев, не затрагивая кристалл полупроводника, что обеспечивает гладкую, бездефектную поверхность в вытравленном окне. Пленка стравливалась до остаточной толщины менее 50 нм, при которой коэффициент отражение составляет более $25 \%$. Это сопоставимо с коэффициентом отражения чистого полупроводника, поэтому наличие остаточного слоя не критично для работы лазера. Стравливание очень небольшого объема материала позволило получить высокую скорость травления. Технологичность и воспроизводимость подхода позволила использовать его для создания серии экспериментальных лазеров с пространственной модуляцией внешних потерь.

\section{4. Селекция горизонтальных мод в лазерах с пространственной модификацией оптических потерь на выход}

Для сравнительного исследования нами были изготовлены три типа лазеров: референсные со стандарт- 
ными зеркалами (тип $A$ ), с просветляющим покрытием (тип $B$ ), с просветляющим покрытием с вытравленным окном с относительной шириной 0.5 (тип $C$ ). Измерения лазеров проводились при комнатной температуре, при накачке постоянным током для подавления эффектов динамического переключения мод. Нанесение просветляющего покрытия увеличивает внешние потери, что увеличивает пороговый ток и дифференциальную квантовую эффективность. Вытравливание окна в просветляющем покрытии увеличивает эффективный коэффициент отражения и соответственно уменьшает внешние потери. Исследование ватт-амперных характеристик лазеров одинаковой длины (рис. 4) не выявило какого-либо негативного влияния окон в диэлектрических покрытиях. В целом изменения порогов и дифференциальной эффективности для всех трех типов лазеров связаны только с изменением коэффициентов отражения зеркал.

Спектр лазеров типа $C$ расщепляется на две ярко выраженные спектральные группы (рис. 5), расстояние между которыми составляет $\sim 20$ нм. В лазерах на квантовых точках подобный эффект, обусловленный широким спектром усиления, наблюдался и ранее [10], причем разным спектральным группам соответствовали разные поперечные горизонтальные оптические моды. Мы исследовали диаграммы направленности со спектральным разрешением, сканируя дальнее поле излучения лазеров оптическим волокном диаметром 200 мкм, соединенным с монохроматором. Измерения проводились в спектральном окне 0.5 нм при фиксированных длинах волны излучения. В пределах одной спектральной группы картины дальнего поля не изменялись (см. вставки на рис. 5) и практически не зависели от тока накачки. При этом более длинноволновой и более интенсивной спектральной группе вблизи 1265 нм соответствует дальнее поле с одним максимумом, а более коротковолновой вблизи $1245 \mathrm{Hм}$ - дальнее поле с тремя максимумами. Асимметричность горизонтального дальнего поля может быть обусловлена асимметрией полоскового волновода и вытравленного окна, а также неравномерным растеканием тока.

Мы выполнили численное двумерное моделирование оптических мод исследуемых лазеров, учитывающее вертикальный волновод и профиль полоскового волновода, с помощью программного пакета FIMMWAVE (Photon Design), которое показало, что в лазерах данной геометрии с однородным выходным зеркалом могут существовать четыре поперечные горизонтальные моды. Дальнее поле коротковолновой спектральной группы хорошо аппроксимируется суперпозицией нулевой, второй и третьей мод с преобладанием второй моды. Дальнее поле длинноволновой группы практически полностью состоит из нулевой моды с небольшим вкладом третьей моды. Суммарная доля интенсивности излучения, приходящаяся на фундаментальную моду, составляет более 70\%. Характерной особенностью модового состава является отсутствие излучения моды 1-го порядка, которая полностью подавлена за счет использования просветляю-

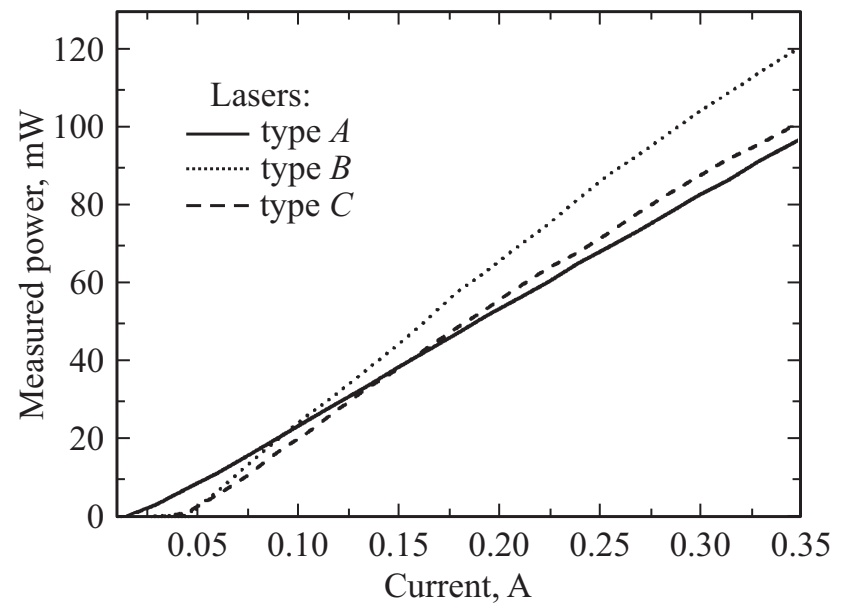

Рис. 4. Зависимость свет-ток для лазеров без покрытий (тип $A$ ), лазеров с напыленным просветляющим покрытием (тип $B$ ) и лазеров с частично вытравленным просветляющим покрытием (тип $C$ ). Длина резонатора 2 мм, ширина полоскового волновода 10 мкм.

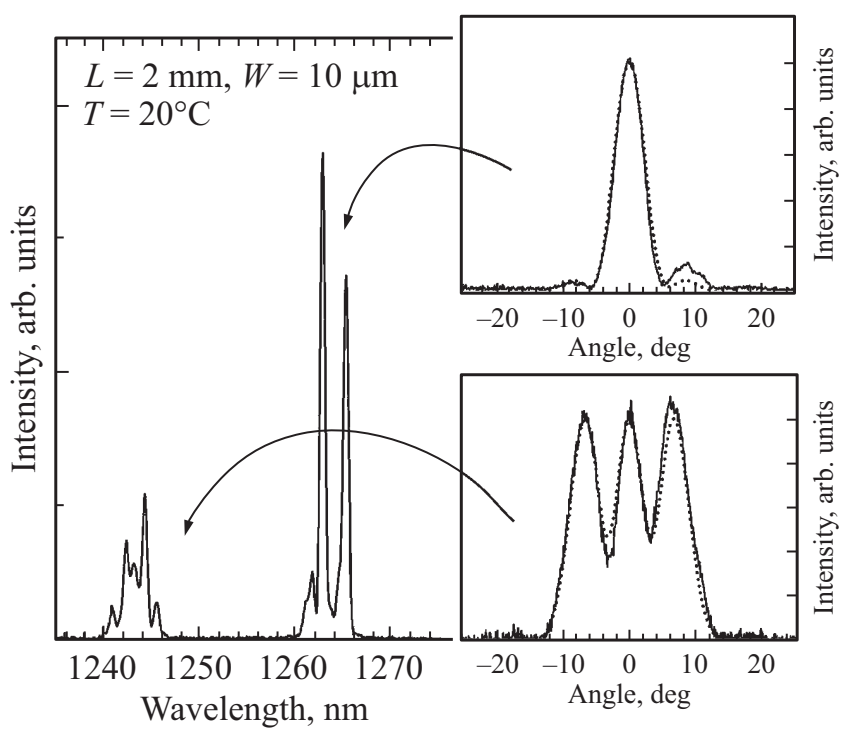

Рис. 5. Спектр излучения лазеров с шириной полоскового волновода 10 мкм с пространственной модификацией отражения на зеркале. На вставках показаны диаграммы направленности, соответствующие спектральным группам (сплошная линия эксперимент, пунктирная линия — моделирование).

щего покрытия с вытравленным окном. Оптимизация ширины вытравленного окна и его позиционирования относительно полоскового волновода позволит подавить лазерную генерацию также и на второй моде.

Мы полагаем, что предложенный способ пространственной модификации коэффициента отражения не ограничивается селекцией поперечных мод и может быть использован, например, в массивах оптически связанных одномодовых лазеров [10] для исследования динамических и когерентных эффектов. 


\section{5. Заключение}

Разработана численная модель селективного изменения внешних оптических потерь поперечных горизонтальных мод полосковых полупроводниковых лазеров, основанная на пространственной модуляции коэффициента отражения выходного зеркала. Показано, что разница в оптических потерях для мод разного порядка может достигать величин более $3 \mathrm{~cm}^{-1}$, что делает возможным выборочно подавлять оптические моды. В качестве тестовых образцов были изготовлены лазеры с активной областью на основе 10 слоев InAs/InGaAs-квантовых точек с многомодовым полосковым волноводом шириной 10 мкм. Для экспериментального подтверждения эффекта подавления поперечных мод был разработан лабораторный технологический метод пространственной модуляции внешних потерь, основанный на напылении просветляющей диэлектрической пленки $\mathrm{Ta}_{2} \mathrm{O}_{3}$ и последующего прецизионного вытравливания в ней окна фокусированным ионным пучком. Ориентация пучка параллельно лазерному зеркалу позволила избежать повреждения его поверхности. Анализ ватт-амперных характеристик лазеров показал отсутствие негативного влияния окон в просветляющем покрытии на пороговый ток и эффективность излучения. Окно с относительной шириной 0.5 позволило полностью подавить лазерную генерацию на поперечной горизонтальной моде 1-го порядка. Численное моделирование показало возможность селективного подавления нескольких поперечных мод высокого порядка, что может быть экспериментально реализовано при оптимизации ширины и геометрического расположения вытравленного окна.

Работа выполнена при частичной поддержке гранта Российского фонда фундаментальных исследований (проект 16-29-03279).

\section{Список литературы}

[1] P. Crump, J. Decker, M. Winterfeldt, J. Fricke, A. Maasdorf, G. Erbert, G. Trankle. Proc. SPIE High-Power Diode Laser Technology and Applications XIII, 9348, 93480D (2015).

[2] J.-K. Chen, S.-C. Lee. IEEE J. Quant. Electron., 23 (8), 1283 (1987).

[3] O.P. Kowalski, M. Silver, Q. Bocang, S. McMaster, D.A. Yanson, S.D. McDougall, J.H. Marsh. Proc. Int. Conf. Indium Phosphide and Related Mater. (Glasgow, Scotland, UK, 547, 2005).

[4] M. Szymanski, J.M. Kubica, P. Szczepanski, B. Mroziewicz. J. Phys. D: Appl. Phys., 30 (8), 1181 (1997).

[5] D. Stryckman, G. Rousseau, M. D'Auteuil, N. McCarthy. Appl. Optics, 35 (30), 5955 (1996).

[6] J.P. Wittke, I. Ladany. J. Appl. Phys., 48 (7), 3122 (1977).

[7] C.A. Volkert, A.M. Minor. MRS Bull., 32 (5), 389 (2007).

[8] G.R. Nash, J.L. Stokes, J.R. Pugh, S.J.B. Przeslak, P.J. Heard, J.G. Rarity, M.J. Cryan. Appl. Phys. Lett., 100 (1), 011103 (2012).
[9] A.R. Kovsh, N.A. Maleev, A.E. Zhukov, S.S. Mikhrin, A.P. Vasil'ev, Y.M. Shernyakov, M.V. Maximov, D.A. Livshits, V.M. Ustinov, Z.I. Alferov, N.N. Ledentsov, D. Bimberg. Electron. Lett., 38 (19), 1104 (2002).

[10] L. Harris, D.J. Mowbray, M.S. Skolnick, M. Hopkinson, G. Hill. Appl. Phys. Lett., 73 (7), 969 (1998).

Редактор Г.А. Оганесян

\section{Lateral mode discrimination in edge-emitting lasers with spatially modulated facet reflectivity}

N.Yu. Gordeev' ${ }^{1}$, A.S. Payusov' 1 , I.S. Mukhin ${ }^{2,3}$, A.A. Serin'1, M.M. Kulagina1', Yu.A. Guseva', Yu.M. Shernyakov' ${ }^{1}$ Yu.M. Zadiranov ${ }^{1}$, M.V. Maximov ${ }^{2}$

${ }^{1}$ loffe Institute, 194021 St. Petersburg, Russia

2 St. Petersburg Academic University, 194021 St. Petersburg, Russia

3 ITMO University, 197101 St. Petersburg, Russia

Abstract A post-growth technique aimed at spatial modification of facet reflectivity of edge-emitting diode lasers has been proposed. It is based on the deposition of an antireflection coating and subsequent precise etching by a focused ion beam. The technique allowed suppressing of high-order lateral modes in $10 \mu \mathrm{m}$ stripe lasers based on ten layers of InAs/InGaAs quantum dots. 